\title{
Can undernutrition predict length of stay in patients admitted to a short-stay acute care unit?
}

\author{
M. Finn \\ Department of Nutrition \& Dietetics, Adelaide \& Meath Hospital, Dublin 24, Republic of Ireland
}

Undernutrition may be associated with increased morbidity, length of stay (LOS), complication rates, hospital re-admission rates and, subsequently, higher hospital and rehabilitation costs ${ }^{(1)}$. This university hospital contains an acute care unit with a proposed maximum LOS of approximately $5 \mathrm{~d}$. This thirty-nine-bed unit employs one whole-time dietitian.

The primary aim of the present pilot study was to determine the relationship between nutritional status and hospital LOS. The study also looked at whether living situation before admission and serum albumin levels on admission could predict LOS.

During a 2-month period nutritional assessment was performed on fifty-one randomly-selected patients within $48 \mathrm{~h}$ of admission. Undernutrition was defined as: (a) BMI $<18.5 \mathrm{~kg} / \mathrm{m}^{2}$; or (b) BMI $<20 \mathrm{~kg} / \mathrm{m}^{2}$ with co-existing triceps skinfold thickness (TSF) or mid-arm muscle circumference $<15$ th percentile ${ }^{(2)}$. Percentage weight loss was also calculated. Each patient was screened using three different screening tools (malnutrition universal screening tool, malnutrition screening tool and subjective global assessment (SGA)). LOS and information on living situation before admission were also recorded. Regression analysis was carried out using Minitab statistical package (Minitab Inc., State College, PA, USA).

Median LOS was $6 \mathrm{~d}$. There was a trend towards a longer LOS among the undernourished group as identified by SGA (mean $10 \mathrm{~d} v$. $7.2 \mathrm{~d}$; median $7 \mathrm{~d} v .6 \mathrm{~d}$ ). There was also a weak association between percentage weight loss and LOS.

Only $14 \%$ of patients lived alone before admission. Those living alone were significantly older than those living with a spouse or sibling (70 years $v .59$ years; $P=0.018$ ). There was a trend towards a longer LOS (mean $12.7 \mathrm{~d} v .7 .7 \mathrm{~d}$; median $7 \mathrm{~d} v .6 \mathrm{~d}$ ), lower weight (mean $69.6 \mathrm{~kg} v .76 .8 \mathrm{~kg}$ ) and lower TSF (mean $3.71 \mathrm{~mm} v .5 .30 \mathrm{~mm}$ ) among those who lived alone.

Albumin levels were normal $(35-38 \mathrm{~g} / \mathrm{l})$ in $73 \%$ of cases, regardless of nutritional status. However, there was a trend towards a lower albumin level among the undernourished patients as identified by SGA $(38.7 \mathrm{~g} / 1$ v. $40.3 \mathrm{~g} / \mathrm{l})$. There was no association between LOS and albumin $(P=0.746)$. Finally there was a significant negative correlation between $\mathrm{C}$-reactive protein and serum albumin $(P=0.063)$.

The present small pilot study indicates that patient age, living situation and extent of undernutrition may predict LOS in an acute care unit. Longer LOS has major healthcare cost implications; thus, timely assessment of nutritional status is important. Rather than being a reliable nutritional indicator, serum albumin is linked to inflammatory status. Further research is warranted with larger numbers.

1. Pichard C, Kyle UG, Morabia A et al. (2004) Am J Clin Nutr 79, 613-618.

2. Planas M, Audivert S, Perez-Portabella C et al. (2004) Clin Nutr 23, 1016-1024. 